S. O'Byrne · P.M. Danehy · A.F.P. Houwing

\title{
Investigation of Hypersonic Nozzle Flow Uniformity Using NO Fluorescence
}

Received: date / Revised version: date

\section{Introduction}

Testing and validation of flowfield computations are important and increasingly common uses for ground-test experiments in hypersonic facilities. Such experiments have the potential to provide a controlled and well-understood flow to test computations. Ground-test facilities are only useful for such comparisons when the flows they produce are uniform and repeatable. Variations in the flow properties at the exit of a hypersonic nozzle can cause significant errors in interpreting complex flows. This is particularly true for supersonic combusting flows, which are very sensitive to variations in temperature and freestream composition.

The experiments described in this paper follow the experiments of Palma et al. [13], in which planar laser-induced fluorescence (PLIF) of nitric oxide (NO) was used to measure both vibrational and rotational temperatures in the same facility as in the current work, at slightly different freestream flow conditions. Palma et al. demonstrated good agreement between measured rotational temperature and a nonequilibrium one-dimensional nozzle calculation. The measured vibrational temperatures were higher than the computed value, although they exhibited the expected vibrational freezing behaviour. This disagreement was attributed to nonlinearities in the imaging system and nonuniformity in the flow. The nonuniformities were assumed to be due to contamination by driver gas, and were found in $32 \%$ of the images.

\section{Send offprint requests to: Sean O'Byrne}

\section{S. O’Byrne}

University of New South Wales, Australian Defence Force Academy, Canberra, Australia, 2600

Tel.: +61-2-6268-8353

Fax: +61-2-6268-8276

E-mail: s.obyrne@adfa.edu.au

P.M. Danehy

Advanced Sensing and Optical Measurement Branch, MS 493, NASA Langley Research Center, Hampton, VA, USA, 23681.

A.F.P. Houwing

Department of Physics and Theoretical Physics, Australian National University, Canberra, Australia 0200.
In this paper we use nitric oxide PLIF to visualise flow at the exit of a hypersonic conical nozzle, to determine operating conditions that would allow more uniform nozzle flow than that of Ref. [13], and to explain the mechanism most likely to be responsible for the nonuniform flow. PLIF is well established as a versatile diagnostic technique and has been used to measure rotational temperature $[8,13]$, velocity $[4,3]$, mole fraction $[15,5]$ and pressure [7] in a variety of combustion and hypersonic flow fields. It is particularly well suited to free-piston shock tunnel facilities because nitric oxide is produced in the plenum of the facility, removing the need for external seeding of the flow.

\section{Flow facility and conditions}

The experiments were performed in the $\mathrm{T} 2$ free-piston shock tunnel facility at the Australian National University [17]. This is a small shock-tunnel facility with a run-to-run turnaround time of less than an hour, making it ideal for parametric studies like the present one that require a large number of tunnel runs to produce a conclusive result.

Free-piston shock tunnels are impulse facilities that can generate the pressures and stagnation enthalpies required for simulation of hypersonic atmospheric re-entry flows [6]. In these facilities, a piston is used to compress a driver gas to pressures high enough to rupture a steel diaphragm separating the driver gas from the test gas. This generates a shock wave that propagates through the test gas in the shock tube and reflects at the end-wall of the tube, producing a region of high pressure and temperature that acts as the reservoir for a converging-diverging hypersonic nozzle.

The beginning and end of the facility's test time is dictated by the time required to start the nozzle flow and the arrival of the driver gas at the nozzle exit, respectively. The nozzle starting time can be inferred from the ratio of pitot pressure measured at the nozzle exit to the pressure in the nozzle reservoir. For these experiments, the nozzle starting process was complete $150 \mu$ s after the reflection of the incident shock at the end-wall of the facility. The arrival of driver gas is more difficult to determine because it is not usually 


\begin{tabular}{llll}
\hline \hline Fill conditions & Mixture & $\begin{array}{l}p \\
(\mathrm{kPa})\end{array}$ & $\begin{array}{l}T \\
(\mathrm{~K})\end{array}$ \\
\hline Test gas & $1.1 \% \mathrm{O}_{2}+98.9 \% \mathrm{~N}_{2}$ & 100 & 297 \\
Driver gas & $31.3 \% \mathrm{Ar}+68.7 \% \mathrm{He}$ & 118.6 & 297 \\
\hline \hline
\end{tabular}

\begin{tabular}{lllll}
\hline \hline $\begin{array}{l}\text { Reservoir } \\
\text { conditions }\end{array}$ & $\begin{array}{l}p_{0} \\
(\mathrm{MPa})\end{array}$ & $\begin{array}{l}T_{0} \\
(\mathrm{~K})\end{array}$ & $\begin{array}{l}H_{0} \\
(\mathrm{MJ} / \mathrm{kg})\end{array}$ & $\begin{array}{l}u_{s} \\
(\mathrm{~m} / \mathrm{s})\end{array}$ \\
\hline Value & 27.5 & 4590 & 5.8 & 2400 \\
Uncertainty $(\%)$ & 5.1 & 3.9 & 3.4 & 2.1 \\
\hline \hline
\end{tabular}

\begin{tabular}{llllll}
\hline \hline $\begin{array}{l}\text { Mid-sheet } \\
\text { conditions }\end{array}$ & $\begin{array}{l}p_{\infty} \\
(\mathrm{kPa})\end{array}$ & $\begin{array}{l}T_{\infty} \\
(\mathrm{K})\end{array}$ & $\begin{array}{l}u_{\infty} \\
(\mathrm{m} / \mathrm{s})\end{array}$ & $\begin{array}{l}\rho_{\infty} \\
\left(\mathrm{kg} / \mathrm{m}^{3}\right)\end{array}$ & $M_{\infty}$ \\
\hline Value & 2.8 & 365 & 3076 & 0.026 & 7.90 \\
Uncertainty (\%) & 7.0 & 7.0 & 2.5 & 1.5 & 1.5 \\
\hline \hline
\end{tabular}

Table 1 Baseline T2 facility operating conditions for the experiments.

accompanied by large changes in static pressure, pitot pressure or heat flux. Several techniques have been developed to determine the time of arrival of the driver gas, the most successful and commonly adopted of which use the change in shock angle on a wedge caused by the different ratio of specific heats for the diatomic test gas and the monatomic driver gas $[18,14]$. These techniques work well in large facilities, but are more difficult to implement and less precise in a small nozzle such as that of T2, shown in Fig. 1. An optical device that senses shock-layer thickness upstream of a blunt body has been used [11] in the T2 facility at similar operating conditions to those used in these tests. Measurements with this device indicate a difference in the flow luminosity between 400 and $500 \mu \mathrm{s}$ after shock reflection in this facility, although it could not be conclusively proved that this was entirely due to driver-gas arrival. PLIF images are therefore typically acquired $350 \mu \mathrm{s}$ after shock reflection in this facility. Previous measurements have shown that the shock waves and separated region in a near-wake flow will establish a steady state within $300-350 \mu$ s of shock reflection at these freestream conditions [12].

The test gas used for these experiments was a mixture of $1.1 \%$ oxygen in nitrogen. The shock reflection process dissociates some of the oxygen, forming approximately $1 \% \mathrm{ni}-$ tric oxide in the reservoir, and that composition freezes near the throat of the nozzle, producing NO at the nozzle exit that is used for the fluorescence imaging. The $1.1 \%$ mixture is used rather than air to reduce the influence of both absorption of the laser sheet across the nozzle and interfering fluorescence from $\mathrm{O}_{2}$ in the Schumann-Runge bands.

The initial facility fill conditions, nozzle-reservoir conditions and nozzle-exit conditions for the experiments are shown in Table 1, and serve as a baseline condition for the tests. The uncertainties in Table 1 were determined from the uncertainties in the nozzle expansion angle, shock speed and the nozzle-reservoir pressure. Calculations were performed at \pm 1 standard deviation of these properties, and the propagation of the measurement uncertainties through the calculation was used to estimate the uncertainty in nozzle-exit properties. The measured shock speed in the shock tube and the measured nozzle-reservoir pressure were used in an equilibrium shock tube calculation [9] to compute the stagnation enthalpy of the condition. A one-dimensional time-marching inviscid vibrational nonequilibrium nozzle code based upon Ref. [1] was used to determine the nozzle exit conditions. Matching the calculated pitot pressure at the nozzle exit with measurements was used to account for the nozzle boundary layer displacement thickness in the inviscid calculations.

The nozzle used for the experiments was a $7.5^{\circ}$ halfangle conical nozzle, with reservoir and throat regions as shown in Fig. 1. The throat of the nozzle is an insert that also serves to hold in place a mylar diaphragm, shown in the figure. The diaphragm initially separates the test gas in the shock tube from the test section and nozzle, which are maintained at vacuum. The diaphragm bursts as the shock propagates through the throat. Two nozzle-throat inserts were fabricated: one with a converging conical end-wall, having a half-angle of $30^{\circ}$ and the other with a flat end-wall, as shown by the dashed line in the figure.

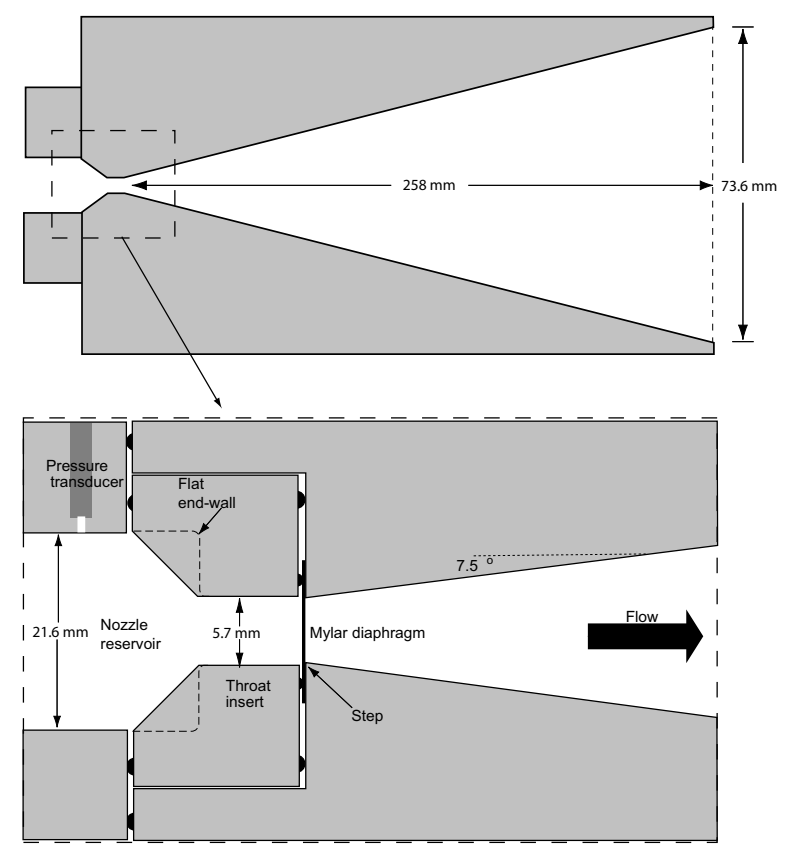

Fig. 1 Schematic of the nozzle-throat region

\section{PLIF and flow uniformity}

The NO PLIF signal intensity depends upon a variety of flowfield and laser-based parameters [16]. For simplicity, the excitation and fluorescence processes can be modeled as being limited to the initial and excited states of the molecule, 
denoted using the labels 1 and 2 . The measured signal $S_{f}$ arising from a region of the laser sheet imaged onto a camera is then described by

$S_{f}=C E_{p} g\left(v_{\|}, T, p\right) f_{B}(T) \chi n B_{12}\left[\frac{A_{21}}{A_{21}+Q_{21}(p, T)}\right]$

where $C$ is a constant incorporating the detection efficiency of the optics, $E_{p}$ is the pulse energy of the laser, $g$ is the convolution of the laser and transition linewidth, $v_{\|}$is the component of the flow velocity in the direction of laser propagation, $f_{B}$ is the Boltzmann fraction in the initial state, $\chi$ is the mole fraction of $\mathrm{NO}$, and $n$ is the total number density. $A$ and $B$ are the Einstein coefficients for spontaneous emission and stimulated absorption, respectively. $Q$ is the rate of collisional quenching and the term in square brackets is the fluorescence yield, a measure of the proportion of the excited molecules that fluoresce. Variations in the flowfield temperature, pressure, velocity and NO mole fraction influence the signal through $f_{B}, \chi, Q_{21}$ and $v_{\|}$, which can complicate the interpretation of images in some flows. The nozzle flow is a simple environment in this regard, as the mole fraction should be uniform throughout the flowfield and there should be no significant variations across the radial extent of the core flow in either temperature or pressure. The conical shape of the nozzle means that the flow expands in the downstream axial direction, but across the region of interest the variation in pressure, temperature and velocity amounts to $\pm 20,5$ and $0.2 \%$ of the mean values, respectively. Modelling of the fluorescence based upon Eq. 1 indicates that none of these variations should change the PLIF signal by more than $\pm 1.1 \%$ in the axial direction. The component of velocity in the radial direction is also small enough to have a negligible effect on the PLIF signal.

\subsection{PLIF apparatus}

The PLIF system used in these experiments was the same as that used in Ref. [13]. It consisted of an excimer laser (Lambda Physik EMG150ETS) pumping a Lambda Physik Scanmate 2 dye laser, frequency doubled to $226 \mathrm{~nm}$. The operating wavelength was determined by scanning the wavelength across the NO transitions in a flame and imaging the fluorescence on a spectrometer. One aspect in which this experiment differs from the previous one is that the images were obtained using a Princeton Instruments PI-ICCD gated, intensified CCD camera. The camera was calibrated using known concentrations of NO in a gas cell, to ensure that both the camera response and the fluorescence varied linearly with laser energy. Measurements in a low-pressure cell indicated a spectral width of 0.18 wavenumber for the laser line. The laser sheet, formed using a cylindrical and a spherical lens, was $0.7 \pm 0.05-\mathrm{mm}$ thick at the mid-point of the measurement location. The intensifier of the camera was integrated over a 100-ns period, sufficient to capture most of the available fluorescence without significantly blurring the image. The fluorescence was spectrally filtered from flow luminosity and from Mie scattering using a Schott UG5 ab- sorption filter. The laser sheet was located between 20 and $60 \mathrm{~mm}$ downstream of the nozzle exit, as shown in Fig. 2. This image was obtained with the laser tuned off the transition, and the signal that can be seen is due to Mie scattering by particles in the flow, particularly in the nozzle boundary layer. The scatter intensity is 100-200 times less intense than the PLIF signal intensity. The PLIF images were obtained within the core flow of the nozzle, in the region indicated by the dashed box.

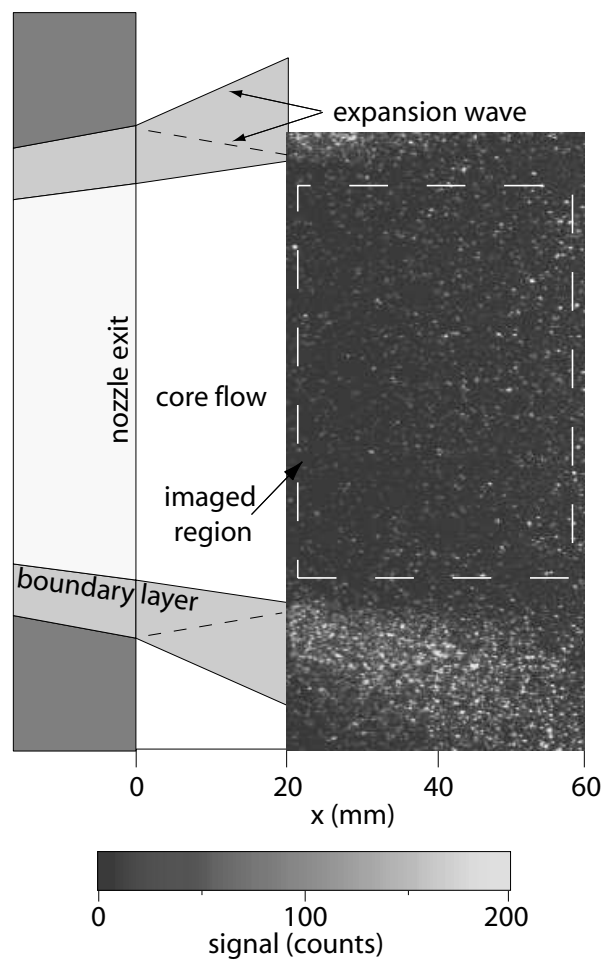

Fig. 2 Scatter image showing the measurement region and the extent of the nozzle core flow. The dashed box shows the region imaged in subsequent figures in this paper.

The ${ }^{O} P_{12}$ (2.5) transition of the $A^{2} \Sigma^{+} \leftarrow X^{2} \Pi(0,0)$ band of NO was used for the measurement, and was chosen because it is a relatively weak, isolated transition that suffers minimal interference from neighbouring transitions: calculations indicate that approximately $0.1 \%$ of the PLIF signal arises from neighbouring transitions at these conditions. The laser energy at the test section was $350 \mu \mathrm{J}$, distributed across the laser sheet. This corresponds to less than $10 \%$ of the saturation irradiance, as measured using a static gas cell at the expected freestream pressure. The irradiance was kept low to ensure that the PLIF signal varied linearly with laser energy. The images were corrected for spatial intensity variations by normalising the signal to the signal measured in a concentrated rhodamine 6G solution in a dye cell. 


\section{Experiments}

Figure 3 shows how PLIF identifies nonuniform flow in the shock tunnel. We define a uniform image as one for which the standard deviation of the signal is less than than $10 \%$ of the average value in the region of interest. The last of the images satisfies this criterion and was considered uniform, while the others were considered nonuniform. Significant variation in peak PLIF signal is evident between the nonuniform images. The nonuniformity manifests as irregularly shaped regions of low signal, some of which are large on the scale of the nozzle flow. As the images are obtained in a planar region, the nonuniformities are not averaged out along the line of sight, and are clearly shown. There was no repeatable pattern of signal distribution from one tunnel run to the next. All the uniform images had average signal intensities between 30000 and 40000 counts, while all the nonuniform images had significantly lower average intensities. These images are qualitatively similar to those presented in Palma et al. [13].

It should also be noted that the nonuniformities that are readily apparent in Fig. 3 were not detected by surface heat flux measurements, shadowgraph images or pitot pressure measurements. PLIF, being species-specific and sensitive to several flow properties, is an excellent means of detecting nonuniformity in ostensibly uniform flow.

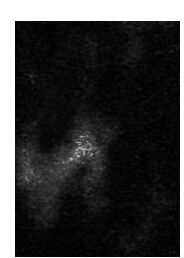

$\max =4000$

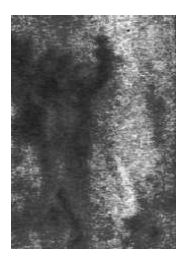

$\max =18000$

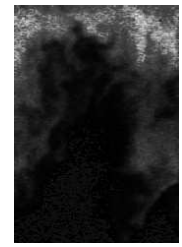

$\max =18000$

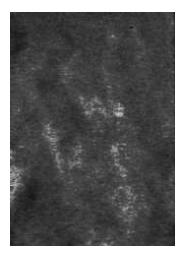

$\max =10000$

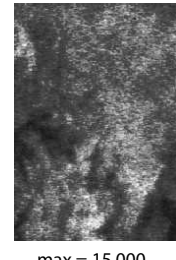

$\max =15000$

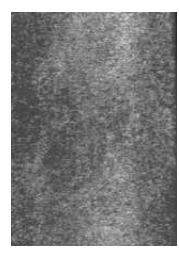

$\max =40000$

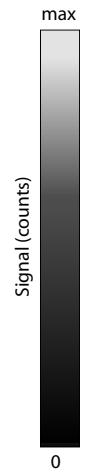

Fig. 3 The first five images indicate the variety of nonuniform PLIF images obtained, while the sixth image (bottom right) is representative of an image that was considered uniform. Each image uses a linear colour table with zero minimum counts and the maximum scaled to the number beneath the image.

Initial tests, performed after the results described in [13] and with the aim of eliminating flow nonuniformities, involved reducing the nozzle throat diameter from $6.35 \mathrm{~mm}$ to $5.7 \mathrm{~mm}$. This reduces the mass flux through the nozzle and would increase the time before driver gas reached the nozzle exit. If driver-gas contamination were the cause of the problem, an increase in the proportion of uncontaminated images would be expected at the same acquisition time.
The result of the reduction in throat size between the previous PLIF experiments and these was that the occurrence of nonuniform flow increased from $32 \%$ to $66 \%$ rather than decreasing. Even using only the uniform images, as defined above, the run-to-run variations in the mean of normalised PLIF signal in the 150 by 250-pixel imaged region were approximately $25 \%$. Measurements performed at the expected freestream pressure in an atmospheric-temperature gas cell showed pulse-to-pulse variations in the normalised PLIF signal, due to variations in laser mode structure, of $15 \%$. Significant numbers of tunnel runs were being wasted and the validity of quantitative measurements obtained after discarding a high proportion of the images was open to question, so a series of experiments was planned to determine the cause of the nonuniform PLIF signal. These experiments varied several parameters, one at a time, that might have been responsible for the nonuniformity in the images. Each change was discarded after at least five tunnel runs if there was no obvious improvement in the signal quality. The following changes were examined:

- Seeding of the driver gas with NO. Tests were performed using a freestream of pure nitrogen and seeding the driver gas with $1 \% \mathrm{NO}$ by volume, performing NO PLIF at successively longer delays to determine the time and location of driver gas as it arrived at the nozzle exit. No PLIF signal could be detected in these tests, despite using delays of as much as $800 \mu$ s after shock reflection before acquiring images. This was considered an inconclusive result, as the NO in the driver gas may have dissociated during the piston compression process.

- Time of image acquisition. The time after shock reflection at which images were obtained was varied, acquiring images at less than $350 \mu$ s after shock reflection. If the problems were caused by the premature arrival of driver gas, the nonuniform flow regions should be less apparent at earlier delay times. No dependence on the acquisition time was noticeable, with visible nonuniformity apparent even $150 \mu$ s after shock reflection.

- Shock tube fill pressure. Increasing the shock tube fill pressure has the effect of varying the shock speed and hence the stagnation enthalpy of the test gas. Lowerenthalpy conditions tend to have a longer test time, and therefore less driver gas would be expected to be present at the test time, than for a higher-enthalpy condition. Tests were performed at a shock tube fill pressure of $150 \mathrm{kPa}$, decreasing the shock speed and hence decreasing the enthalpy to $4.5 \mathrm{MJ} / \mathrm{kg}$. This decrease in stagnation enthalpy had no discernible effect on the flow uniformity.

- Shape of the shock tube end-wall. The nozzle end-wall for the T2 facility consisted of a conical converging section, as shown in Fig. 1. A replacement insert with a flat end-wall was tested in place of the $30^{\circ}$ converging section and is shown by the dashed lines in Fig. 1. This change was made to test whether the end-wall geometry influenced the conditions in the nozzle reservoir. Previous work shows that the interaction of the reflected shock 
with the boundary layer on the shock tube wall can generate recirculating flow [2], and that the interaction of the reflected shock with the contact surface separating the test gas from the driver gas can cause premature jetting of the driver gas into the test gas [10]. Either of these two effects could generate large variations in temperature and composition in the flow entering the nozzle. Experimental images showed nonuniform freestream flow using both end-wall configurations.

- Driver-gas composition. Experiments performed using one of the driver-gas-contamination detectors mentioned above have shown that the onset of driver-gas contamination may be delayed by causing the condition to be slightly under-tailored [18]. This was achieved in our experiments by increasing the proportion of helium to argon in the compression tube to $80 \% / 20 \%$, while keeping the pressure of the mixture constant. Although tests were only performed at the nominal $350-\mu$ test time, freestream nonuniformities were still evident in most images.

- Diaphragm burst pressure. A tailored operating condition was found at a higher diaphragm burst pressure of $58.5 \mathrm{MPa}$, compared with the baseline diaphragm burst pressure of $46.9 \mathrm{MPa}$, to determine the effect of increasing the reservoir pressure to $30.3 \mathrm{MPa}$. This change also did not discernibly effect the likelihood of nonuniform flow at the nozzle exit.

- Test gas composition. Experiments were performed using air as the test gas rather than the $1 \% \mathrm{O}_{2}$ in $\mathrm{N}_{2}$ mixture, to determine whether the uniformity of the gas was related to the amount of NO in the mixture. All tests performed using air as the test gas were uniform according to the previously described criterion.

Therefore, the only one of the above changes that had a demonstrable and repeatable effect on the flow uniformity was changing the test gas to air. For those tests, all six of the images were uniform. This positive finding, combined with the negative results for all the other tests, indicates strongly that the nonuniform flow is not caused by premature drivergas contamination. If driver gas were the problem, it would interfere with the air as it did with the $1 \% \mathrm{O}_{2}$ in $\mathrm{N}_{2}$ mixture, showing a similar percentage decrease in signal. The fact that the dark regions could be seen for the $1 \% \mathrm{O}_{2}$ in $\mathrm{N}_{2}$ mixture regardless of when the images were acquired during the test time also indicates that the nonuniformity is not caused by driver-gas contamination.

Another possible cause of the nonuniform flow is the production of soot. The shock tunnel produces a fine black soot, caused by decomposition of the molybdenum grease on the piston o-rings, residue from the kerosene and acetone used to clean the compression tube and material abraded from the piston rings. This soot may absorb the fluorescence. The main inconsistency in this possible explanation is that the laser sheet is never affected in any of the images. Large clouds of particulates blocking the fluorescence would cause the laser sheet to exhibit streaks where the particles scatter or absorb the laser radiation, and this was not apparent in the images.

Upon completion of the tests described above, the 5.7$\mathrm{mm}$ diameter nozzle-throat area was examined closely and erosion of the surface material was found 1.0-1.5 throat diameters downstream of the mylar diaphragm at the nozzle throat. The refurbished throat had been in operation for 267 facility runs. A small $(0.1-0.2 \mathrm{~mm})$ forward-facing step in the nozzle profile was noted at the diaphragm position where the insert meets with the diverging part of the nozzle. The most likely explanation for the erosion is that either the step or the cavity that forms when the mylar diaphragm bursts causes the boundary layer to separate at the throat and reattach at the point where the erosion occurs. This boundarylayer separation may have caused the entrainment of cooler boundary-layer test gas containing less NO into the nozzle flow.

Once the erosion was noticed, the throat was rebored to 7-mm diameter, taking care to ensure that there was no discontinuity between the insert and the nozzle. The largerdiameter throat meant that the diaphragm was located in the region of subsonic flow upstream of the nozzle throat, where it was likely to cause less disturbance to the nozzle flow than at the throat. The erosion near the throat was repaired. Care was also taken to remove any soot from the throat before each tunnel run. These changes resulted in a rate of uniform images of more than $95 \%$ in subsequent tests, where a uniform image is defined as one with signal standard deviation less than $10 \%$ of the mean in the imaged region.

\section{Conclusion}

Considering the results listed above, the most likely reason for the flow nonuniformity shown in Fig. 3 is the entrainment of cooler gas from the boundary layer into the freestream caused by flow separation at the throat. This effect is more important in smaller facilities, where the boundary layer makes up a larger proportion of the total flow through the throat than in large facilities. Perhaps the effect on the air flow is less than on the $1 \% \mathrm{O}_{2}$ in $\mathrm{N}_{2}$ mixture because the mole fraction of $\mathrm{NO}$ in the air flow is still great enough in the boundary-layer flow to produce measurable fluorescence. This is still only supposition, however, and other factors may have contributed to the nonuniformity.

Upon modifying the nozzle throat, images were significantly more uniform and the standard deviation in average signal between tunnel runs reduced from $25 \%$ to $15 \%$. The latter value is consistent with pulse-to-pulse variations in PLIF signal obtained in a static gas cell. Therefore the variation in signal due to the tunnel performance was less than that due to the laser system once the modifications had been made. 


\section{Acknowledgments}

The authors wish to thank Mr. Paul Walsh for his valuable technical assistance with the shock tunnel equipment. They also thank Dr. Philip Palma for providing his LINUS code for calculations and for his valuable contributions to discussions of the PLIF technique. This work was funded by the Australian Research Council.

\section{References}

1. Anderson Jr., J.D.: A time-dependent analysis of vibrational and chemical nonequilibrium nozzle flows. AIAA Journal 8(3), 545550 (1970)

2. Chue, R.S.M., Eitelberg, G.: Studies of the transient flows in highenthalpy shock tunnels. Experiments in Fluids 25(5), 474-486 (1998)

3. Danehy, P.M., Mere, P., Gaston, M.J., O’Byrne, S., Palma, P.C., Houwing, A.F.P.: Fluorescence measurement of the velocity-field produced by the hypersonic separated flow over a cone. AIAA Journal 39(7), 1320-1328 (2001)

4. Danehy, P.M., O’Byrne, S., Houwing, A.F.P.: Flow-tagging velocimetry for hypersonic flows using fluorescence of nitric oxide. AIAA Paper 2001-0302 (2001a)

5. Fox, J.S., Houwing, A.F.P., Danehy, P.M., Gaston, M.J., Mudford, N.R., Gai, S.L.: Mole-fraction-sensitive imaging of hypermixing shear layers. Journal of Propulsion and Power 17(2), 284-292 (2001)

6. Gai, S.L.: Free piston shock tunnels: Developments and capabilities. Progress in the Aerospace Sciences 29, 1-41 (1992)

7. Hiller, B., Hanson, R.K.: Simultaneous planar measurements of velocity and pressure fields in gas flows using laser-induced fluorescence. Applied Optics 27(1), 33-48 (1988)

8. Houwing, A.F.P., Palmer, J.L., Thurber, M.C., Wehe, S.D., Hanson, R.K., Boyce, R.R.: Comparison of planar fluorescence measurements and computational modelling of shock-layer flow. AIAA Journal 34(3), 470-477 (1996)

9. $\mathrm{M}^{\mathrm{C}}$ Intosh, M.K.: Computer program for the numerical calculation of frozen equilibrium conditions in shock tunnels. Tech. rep., Australian National University (1968)

10. Mirels, H.: Shock tube test time limitation due to turbulent-wall boundary layer. AIAA Journal 2(1), 38-54 (1964)

11. Mudford, N.R., O'Byrne, S.: A novel optical driver-gas detector. In: 22nd International Symposium on Shock Waves. Fort Worth, Texas, USA (2001)

12. O'Byrne, S., Houwing, A.F.P.: Establishment of the near-wake flow of a cone and wedge in a transient hypersonic freestream. In: 22nd International Symposium on Shock Waves. Southampton, England (1999)

13. Palma, P.C., Danehy, P.M., Houwing, A.F.P.: Fluorescence imaging of rotational and vibrational temperature in a shock tunnel nozzle flow. AIAA Journal 41(9), 1722-1732 (2003)

14. Paull, A., King, M.D.: A driver gas detection device for shock tunnels. Shock Waves 4(5), 289-291 (1995)

15. Rossman, T., Mungal, M.G., Hanson, R.K.: Nitric-oxide planar laser-induced fluorescence applied to low-pressure hypersonic flow fields for the imaging of mixture fraction. Applied Optics 42(333), 6682-6695 (2003)

16. Seitzman, J.M., Hanson, R.K.: Planar fluorescence imaging in gases. In: Instrumentation for Flows with Combustion. Academic Press Ltd (1993)

17. Stalker, R.J.: A study of the free-piston shock tunnel. AIAA Journal 5(12), 2160-2165 (1967)

18. Sudani, N., Valiferdowski, B., Hornung, H.G.: Test time increase by delaying driver gas contamination for reflected shock tunnels. AIAA Journal 38(9), 1497-1503 (2000) 


\begin{abstract}
Planar laser-induced fluorescence visualisation is used to investigate nonuniformities in the flow of a hypersonic conical nozzle. Possible causes for the nonuniformity are outlined and investigated, and the problem is shown to be due to a small step at the nozzle throat. Entrainment of cold boundary layer gas is postulated as the cause of the signal nonuniformity.
\end{abstract}

Keywords nozzle flow, fluorescence, LIF, nozzle uniformity 